\title{
Consideraciones prácticas sobre las cláusulas de ajuste de precio en contratos de compraventa de acciones*
}

\author{
Ricardo de la Piedra Calle \\ Abogado por la Universidad de Lima. \\ Máster (MBA) por IE Business School.
}

SUMARI0:

I. Introducción.

II. Concepto de cláusulas de ajuste de precio y tipos de ajuste.

III. Formas de valorizar una sociedad.

1. Sobre las expectativas de las partes para determinar el precio.

2. Sobre la valorización.

3. Métodos de valorización de empresas.

IV. Estructuración de cláusulas de ajuste de precio con referencia o corte a la fecha de cierre.

1. Concepto.

2. Tipos de ajuste de precio a la fecha de cierre.

3. El caso especial del locked box.

4. Procedimiento.

V. Estructuración de cláusulas de ajuste de precio por hechos o eventos posteriores al cierre: earnouts.

1. Concepto.

2. Estructuración.

3. Monto del earnout y métodos de valorización.

3.1. Periodo del earnout.

3.2. Forma de pago.

3.3. Tipos de objetivos y metas.

3.4. Cronograma de pagos.

3.5. Integración operacional.

VI. Consideraciones adicionales.

1. Eventual duplicidad con las representaciones y garantías en una cláusula de ajuste de precio.

2. Garantía del pago.

3. Definición de conceptos.

4. Mecanismo de solución de controversias.

v. Conclusiones.

"Money often costs too much" - Ralph Waldo Emerson 


\title{
RESUMEN:
}

Las cláusulas de ajuste precio juegan un rol preponderante en los contratos de compraventa de acciones. Debido a su naturaleza dinámica, las empresas se encuentren en constante movimiento, lo que complica la determinación de su valor y, por ende, su precio. Dependiendo del tipo de ajuste de precio que se pacte, se puede acortar las brechas en la valorización de una empresa entre vendedor y comprador, regular y mitigar los cambios patrimoniales ligados a un cierre diferido o incluso de una valorización hecha con estados financieros desactualizados. Al ser un asunto ligado tanto al tema legal como financiero, entender los conceptos vinculados a su estructuración y ejecución es fundamental para un abogado transaccional.

Palabras Clave: Cláusulas de Ajuste de Precio, Fusiones y Adquisiciones, Valorización de Empresas.

\begin{abstract}
:
Price adjustment clauses play a predominant role in stock purchase agreements. Due to their dynamic nature, companies are in constant movement, which complicates the determination of their value and, therefore, their price. Depending on the agreed type of price adjustment, it can shorten the gaps in the valuation of a company between seller and buyer, regulate and mitigate the changes in assets linked to a deferred closure or even a valuation made with outdated financial statements. Being a matter linked to both legal and financial issues, understanding the concepts related to its structuring and execution is fundamental for a transactional lawyer.
\end{abstract}

Keywords: Price Adjustment Clauses, Mergers and Acquisitions, Company Valuation.

\section{INTRODUCCIÓN}

De acuerdo con la información de J.P. Morgan', durante el año 2017 la actividad global en el mercado de fusiones y adquisiciones -en adelante, "M\&A", por sus siglas en inglés- alcanzó un monto superior a USD 3.7 trillones. Dicha información es consistente con el reporte del Institute of Mergers, Acquisitions and Alliances —en adelante, "IMAA" —, agregándose además, que durante el año pasado se llevaron a cabo más de 50,000 transacciones a nivel mundial.

Al cierre del primer semestre del 2018, el monto global ya había superado los USD 2 trillones ${ }^{2}$, con lo cual la posibilidad de superar los USD 4 trillones proyectada para el $2018^{3}$ parece ser bastante viable.

A nivel Perú, según Transactional Track Record ${ }^{4}$ —en adelante, "TTR" -, al cierre del tercer cuar- to de 2018 se ha realizado actividad en M\&A por USD 6,600 millones, aproximadamente un $10 \%$ adicional que el monto en 2017 a la misma fecha de corte.

En línea con lo anterior, y de acuerdo con la información publicada por Harvard Business Review, vemos que el monto invertido en operaciones de fusiones y adquisiciones se mantiene al alza, pese a que entre el $70 \%$ y $90 \%$ de dichas transacciones no generan los beneficios esperados para el adquirente $-\mathrm{y}$ en algunos casos, no generan beneficios en absoluto-.

En dichas circunstancias, las cláusulas de ajuste de precio han venido jugando en los últimos años un factor preponderante para tratar de dar tranquilidad a las partes, y no detener la ejecución de adquisiciones por discrepancia en el precio -o en la información o método utili-

1. J.P. MORGAN. "2018 Global M\&A Outlook. Navigating consolidation and disruption". Disponible en: < $\underline{\text { https://www. }}$ jpmorgan.com/jpmpdf/1320744801603.pdf>.

2. Mergermarket. "Global \& Regional M\&A Report H1 2018". Disponible en: < https://www.mergermarket.com/info/h12018-global-ma-report-financial-advisor-league-tables $>$.

3. KPMG. "2018 Annual Report. M\&A Predictor". Disponible en: <https://assets.kpmg.com/content/dam/kpmg/xx/ pdf/2018/05/m-and-a-predictor-2018-annual-report.pdf $>$.

4. CHRISTENSEN, Clayton, ALTON Richard. RISING, Curtis y WALDECK, Andrew. "The Big Idea: The New M\&A Playbook". Cambridge, Massachussetts: 2011, Harvard Business Review. 
zado para llegar a definirlo-. Es claro que en las diferentes formas de valorizar una empresa, el vacío que puede existir entre la declaración de un vendedor y la percepción de un comprador, así como las consecuencias en el valor de una empresa en un cierre diferido o en una valorización con estados financieros desactualizados, pueden generar complicaciones en una negociación de M\&A. Por lo que este tipo de cláusulas resultan ideales para lograr cerrar transacciones con divergencias o alguna incertidumbre en la definición del precio entre las partes.

En base a lo expuesto, el objetivo del presente artículo es dar una mirada general de los tipos de cláusulas de ajuste de precio, así como la forma en que se estructuran y en base a qué factores y conceptos financieros y comerciales se aplican.

\section{CONCEPTO DE CLÁUSULAS DE AJUSTE DE PRECIO Y TIPOS DE AJUSTE}

Un estudio de la American Bar Association ${ }^{5}$ demuestra que, durante 2016 y 2017 , el $86 \%$ de los contratos relacionados a M\&A en Estados Unidos - principal referente para la estructuración de acuerdos de adquisición de acciones en nuestro país - contuvo una cláusula de ajuste de precio post-cierre, siendo las principales métricas utilizadas el capital de trabajo, deuda y efectivo.

A nivel conceptual, una cláusula de ajuste de precio establece el mecanismo mediante el cual las partes acuerdan que el precio pactado en el contrato de adquisición de la empresa objetivo se modifique, en caso se verifique con posterioridad al cierre que se han cumplido/incumplido determinadas condiciones o eventos. Si bien estos ajustes se dan con posterioridad al cierre, la fecha de corte para la determinación de estos eventos podría ser (i) a la fecha de cierre - ajuste de precio estándar-; o (ii) con posterioridad a la fecha de cierre - conocido como earnout-. Nada impide que se pacten ambas clases de ajustes, aunque no es lo usual.
La experiencia demuestra que la cláusula de ajuste de precio muchas veces conlleva una problemática operativa. Es que muchas veces abogados y asesores financieros buscan delegar la responsabilidad de la redacción final a la otra. Esto no solo se ve en ciertas negociaciones, sino que fue confirmado por profesionales en cargos financieros decisorios con los que tuve la oportunidad de hacer el MBA. Desde la perspectiva de algunos abogados, al tratarse de una cláusula con contenido financiero, esta debería estar a cargo de la gente de finanzas involucrada en la transacción. Por su parte, los asesores financieros muchas veces prefieren que sean los abogados quienes nos encarguemos de redactar esta sección, para que carguemos con la responsabilidad de asumir cualquier contingencia económica relacionada a ajustes de precio, dado que finalmente esta se incluye en un documento de índole legal - contrato de compraventa de acciones-.

Según adelantamos, las cláusulas de ajuste de precio pueden dividirse en 2 grandes grupos:

a) Cláusulas de ajuste de precio con incidencia o fecha de corte al cierre. Este tipo de cláusulas tienen un rango de aplicación por hechos o eventos ocurridos hasta la fecha de cierre -es decir, con un corte a la fecha de efectividad o materialización de la transacción-. Inclusive si su aplicación se da con posterioridad al cierre - como es usual - tienen un carácter retrospectivo a dicha fecha de corte.

b) Cláusulas de ajuste de precio por hechos o acciones que ocurran con posterioridad a la fecha de cierre. Este tipo de cláusulas basa el ajuste en eventos o condiciones que ocurren con posterioridad a la fecha de cierre, tales como el cumplimiento de ciertas metas o ratios comerciales o financieros. A este tipo de método de ajuste de precio se le conoce como earnouts. 


\section{FORMAS DE VALORIZAR UNA SOCIEDAD}

A efectos de entender qué es y cómo funciona una cláusula de ajuste de precio, creemos conveniente entender en primer lugar cómo se llega a definir el precio de compra de una empresa. Si bien es cierto que un proceso de valorización formal de la sociedad objetivo es un componente crítico para tomar la decisión de llevar a cabo la transacción, esta no es una ciencia exacta, y el monto a pagar no necesariamente se decidirá por el resultado de la valorización.

Así, tenemos que un vendedor podría preferir vender a un precio alto, pero otorgando a cambio un set de declaraciones y garantías extremadamente robustas y un régimen de indemnización beneficioso al comprador; mientras que otro vendedor podría preferir recibir un menor precio por la empresa objetivo, pero tener certeza respecto a que los reclamos derivados de la compraventa serían mínimos en base a lo pactado en el contrato. Lo mismo ocurre con el comprador.

En tal sentido, más allá de los métodos de valorización que explicaremos a continuación, hay que tener en cuenta que el precio final al que un comprador esté dispuesto a adquirir, y un vendedor a transferir, dependerá tanto de la valorización acordada, como de los términos, condiciones de mercado y situación de las partes.

\section{Sobre las expectativas de las partes para determinar el precio.}

Entonces, ¿a qué precio se vende una empresa? El vendedor evidentemente querrá obtener el mayor precio, mientras el comprador querrá obtener la empresa por el menor monto posible. ¿Cómo se amalgama esta situación en la práctica?

Las partes no suelen declarar cuánto vale realmente algo para ellas. No revelan sus motivaciones del todo y muchas veces eso puede generar mayores ganancias para la parte que puede leer mejor los intereses de su contraparte. A manera de ejemplo, un comprador que necesita adquirir una empresa para subsistir en un mercado atomizado estará dispuesto a pagar más por ella que un comprador que simplemente lo hace para adquirir mayor participación de mercado; y esa información usualmente no está disponible para la contraparte -especialmente en la adquisición de empresas privadas-. A saber, una empresa de tecnología innovativa no valdrá igual para una empresa que busca implementar su área de innovación que para un fondo de inversión especializado en empresas de innovación tecnológica, y es labor del vendedor poder identificar el real valor que un comprador está dispuesto a pagar, incluso si esos aspectos no son incluidos como variables en la valorización.

En línea con lo anterior, tenemos que (i) cada comprador valorizará a la empresa objetivo de manera diferente, en base a sus propias motivaciones, necesidades y asunciones y en base a ello planteará un precio; $y$, (ii) desde el punto de vista del vendedor, establecer un precio fijo por la empresa independientemente del tipo de comprador podría generar que la empresa no se venda por el mayor valor ${ }^{6}$.

\section{Sobre la valorización.}

El proceso de valorización de un negocio siempre es realizado por el comprador a efectos de tener certeza en el precio. Además, podría ser realizado (i) por el vendedor - con anterioridad a ofrecer la empresa en venta como parte de un due diligence de vendedor-;y/o, (ii) por un banco de inversión.

El valor de mercado de una empresa puede definirse como el monto en que un comprador y un vendedor acuerdan celebrar la compraventa de una empresa objetivo, sin estar forzados a celebrar la transacción y cumpliendo principios de simetría de información. Ahora bien, esta definición

6. Esta situación usualmente es mitigada a través de una venta por concurso privado, en la que varios postores compiten por hacerse del control de la empresa objetivo. Al estar en un proceso competitivo, usualmente el vendedor asegura recibir el mejor precio posible de parte de los compradores. 
es meramente teórica porque siempre existirán razones que fuercen a alguien a comprar y vender, asimetría informativa a ciertos niveles - por más que estos pueda ser equilibrada entre las partes a través de declaraciones y garantías y el régimen de indemnización-y estrategias de venta. Es por ello que una buena valorización es la piedra angular al momento de definir el precio en una empresa, según describiremos en el punto siguiente.

\section{Métodos de valorización de empresas.}

En el marco de un proceso de adquisición, el comprador y su asesor financiero deben definir de qué forma valorizarán la empresa, a efectos de plantearle un precio al vendedor en base a una metodología que lo sustente. En esa línea, a continuación, describiremos los 5 métodos principalmente utilizados para valorizar una empresa en transacciones de $M \& A$ :

a) Método de flujo de caja descontado: A través de este método, se calculan los flujos de caja de la empresa objetivo hacia el futuro, descontando la tasa de retorno esperada para calcular su valor presente neto. La idea sobre la que se sustenta este método es que, cuando una compañía es transferida, el comprador evidentemente adquirirá también los flujos de caja que genere la operación de la empresa objetivo. Los elementos clave del modelo de valorización bajo comentario son las proyecciones financieras, la definición clara del concepto de flujo de caja y el costo del capital que se utilice para calcular una tasa apropiada de descuento ${ }^{7}$. Los pasos que usualmente sigue el equipo financiero para este tipo de valorización es el siguiente:

(i) El primer paso es desarrollar la proyección de los estados financieros de la empresa objetivo. Como se puede intuir, esto no es tarea fácil, ya que implica tener o adquirir un conocimiento cercano de los resultados históricos de la compañía objetivo, sus operaciones, riesgos asociados y una serie de asunciones relacionadas no solo al objetivo, sino a la industria en la que este desarrolla sus actividades.

(ii) Una vez realizada la proyección, se debe determinar los flujos futuros de la compañía objetivo, esto es, los flujos "libres" que permanecen en la sociedad objetivo luego de deducir todos los gastos y costos relacionados a su actividad. A efectos de claridad, se deben entender como los recursos y flujos que no son necesarios para las operaciones y que, por lo tanto, están listos para ser distribuidos a los accionistas, sin afectar el desarrollo del negocio de la empresa objetivo en el futuro.

(iii) Determinados los montos relacionados a los flujos de caja, se le asigna un valor a futuro en base al horizonte de la proyección -entre 5 y 10 años usualmente, dependiendo del tamaño, industria e información histórica-.

(IV) Obtenido el valor de los flujos a futuro, se debe aplicar una tasa de descuento para traer dicho valor a la actualidad y obtener el valor presente de la empresa objetivo. La lógica es bastante simple: los flujos ocurrirán en el futuro y el valor se ha estimado a futuro, pero como la empresa objetivo se está comprando en el presente, se deben aplicar los principios del valor del dinero en el tiempo, para traer al valor actual la estimación.

(V) Una vez aplicada la tasa de descuento, se obtendrá el valor actual de la compañía objetivo.

b) Método del valor comparable de transacciones y compañías similares: Se basa en el

7. BREALEY, Richard; MYERS, Stewart y ALLEN, Franklin. "Principles of Corporate Finance". Vigésimo-Primera edición. Nueva York: McGraw-Hill Education, 2014, pp. 37-44. 
concepto de utilizar valores comparables de empresas similares a los que se llega mediante la información pública que se encuentre disponible. La racionalidad subyacente es que, al analizar empresas y transacciones similares, se puede inferir la forma y el valor que un vendedor otorgaría a la empresa objetivo. Asimismo, este método se aplica mediante la identificación de transacciones con operaciones similares a las de la empresa objetivo. Definido el valor de la transacción comparable, se puede llegar a un valor para la empresa objetivo.

Una vez determinado el rango de valor, este se ajusta en base a las consideraciones particulares de la empresa objetivo.

Si bien la premisa se puede sustentar teóricamente sin mayor problema, los problemas de aplicación práctica de este método incluyen que (i) las compañías comparables realmente deben reunir las condiciones para ser consideradas como tales; $y$, (ii) debe haber suficiente información disponible respecto a la transacción y a la compañía adquirida, para que el sustento sea veraz. Muchas veces, realizar este método puede arrojar resultados irreales sobre el valor de una compañía.

c) Método de valorización por activos: En supuestos en que los negocios de la compañía dependan principalmente de sus activos fijos, es bastante viable valorizar la compañía a través de sus activos principales, a efectos de determinar el precio 8 .

Al utilizar este método, se deben considerar todos los activos de la empresa objetivo, incluyendo los intangibles. Este último punto puede ser un problema porque, evidentemente, el vendedor tratará de incluir la mayor cantidad de goodwill en la valorización, a efectos de incrementar el precio de la empresa objetivo.

d) Método de análisis de probabilidades: En escenarios en que la información financiera prospectiva de la empresa es compleja y tiene múltiples escenarios que podrían derivar en flujos de cajas totalmente dispares para la empresa objetivo, dependiendo del escenario que se analice, es conveniente utilizar el método de análisis de probabilidades ponderadas para valorizar la empresa. Bajo este método, el valor se basa en los valores actuales de los resultados futuros estimados, pero tomando en cuenta todos los escenarios de la empresa objetivo y asignándoles un peso específico para dicho análisis. Si bien este método es más completo que el de flujo de caja descontado, las dificultades de poder predeciry asignar correctamente el peso a los eventos hacen de este método uno residual ${ }^{9}$.

e) Determinación del precio en base a un múltiplo de EBITDA ${ }^{10}$ : Más allá de la literalidad del acrónimo, el EBITDA es un indicador financiero que mide el beneficio que generan las operaciones de una empresa, independientemente de la forma en que fueron financiadas - ajustando los intereses-, de la tasa de los impuestos, de la manera como los activos fijos se depreciaron y de la amortización de los intangibles ${ }^{11}$.

EI EBITDA es calculado en base al estado de resultados de una compañía, y mide la

8. LIBBY, Robert; LIBBY, Patricia; y SHORT, Daniel. "Financial Accounting, Global Edition”. Séptima edición. Nueva York: McGraw-Hill Education, 2014, pp. 244-251.

9. VAN DER PUTTE, Alexander. “Strategic Foresight: Navigating Uncertainty Course”. IE Business School MBA Course. Madrid: 2016.

10. El EBITDA es un indicador financiero, probablemente el más utilizado. Las siglas derivan de su definición en inglés "earnings before interest, taxes, depreciation, and amortization", esto es, los ingresos de una sociedad antes de intereses, impuestos, depreciaciones y amortizaciones —ingresos antes de la deducción de gastos financieros-.

11. FORSYTH, Juan. "Finanzas empresariales: Rentabilidad y valor". Segunda edición. Lima: 2006, pp. 48-49. 
situación económica de un ejercicio determinado excluyendo los gastos y costos relacionados a la estructura de capital elegida y los impuestos aplicables a la misma.

Bajo este método, las partes acuerdan que el precio de la transacción se determine en base a un múltiplo de EBITDA. Pese a que en principio se trataría de un concepto objetivo, este método siempre ha tenido algo de resistencia porque implica ciertos niveles de subjetividad, lo que conlleva incluso a que la forma de cálculo no es uniforme entre auditoras de primer nivel. Los ejemplos más comunes implican que no todas las empresas reconocen de la misma forma sus ingresos y gastos; o el uso de ingresos y gastos no recurrentes que podrían afectar de manera sustancial el EBITDA de un ejercicio.

Independientemente del método de valorización elegido, es bastante usual que el comprador evalúe también el valor de la empresa objetivo como parte de un grupo económico - de ahora al menos 2 sociedades-, lo cual también dependerá del escenario de integración ${ }^{12}$ en el que nos encontremos. Esto, a efectos de definir el valor estratégico de la compañía, más allá de la valorización de la empresa objetivo como ente independiente. Las asunciones clave que se utilicen para definir el valor estratégico son igual de importantes y deberían ser consistentes con las utilizadas para el método de valorización elegido, a efectos de encontrar las sinergias que establecerán el precio real que el comprador podría pagar por la compañía objetivo ${ }^{13}$.

Pese a que ninguno de los métodos bajo comentario proporcionará un precio que no pueda ser cuestionado de alguna forma, la experiencia demuestra que el método de flujo de caja descontado sigue siendo la forma predilecta de valorizar empresas en el mercado peruano.

\section{ESTRUCTURACIÓN DE CLÁUSULAS DE AJUSTE DE PRECIO CON REFERENCIA O CORTE A LA FECHA DE CIERRE}

\section{Concepto.}

Las cláusulas de ajuste de precio con corte a la fecha de cierre buscan que se pague el precio que realmente corresponde por una compañía, valga la redundancia, a la fecha de cierre.

En la práctica, estas cláusulas sirven para subsanar las eventuales modificaciones entre la fecha de los estados financieros utilizados para la valorización de la empresa/información a la fecha de firma y la fecha de cierre de una transacción. Si bien es cierto que el equipo financiero del comprador utilizará los estados financieros más actualizados que le provean para establecer el valor de la compañía a determinada fecha, inexorablemente habrá un desfase entre la fecha en que se realiza la valorización - basada en estados financieros incluso anteriores-y entre la fecha de cierre de la transacción. Así, se establecerán ciertas métricas a manera de asignar los riesgos entre las partes por las variaciones hasta la fecha efectiva de cierre, definiéndose qué variables corresponden al giro ordinario del negocio de la empresa objetivo y cuáles deben ser motivo de ajuste.

A través de una cláusula de ajuste de precio, con posterioridad al cierre se revisará la información actualizada a la fecha de cierre, con lo cual cualquier variación en los niveles de caja, deuda y capital de trabajo en la empresa objetivo - como hemos mencionado, las principales variables utilizadas para el ajuste de precio-u

12. Existen 2 tipos de integración en las compraventas de empresas: (i) la integración horizontal, que implica adquirir compañías que ofertan bienes o servicios similares a la empresa adquirente con la finalidad de ser más competitivas y adquirir mayor posicionamiento en el mercado en el que operan o abrir nuevos mercados, obteniendo por consecuencia un mayor dominio; y, (ii) la integración vertical, a través de la cual se adquieren compañías que desarrollan alguna de las fases de la cadena de producción del bien o servicio que ofertan, con el propósito de reducir costos, márgenes y estandarizar procesos.

13. HULL, John y SANKARSHAN, Basu. “Options, futures and other derivatives”. Octava Edición. India: 2016, pp. 629-630. 
otro tipo de activos/ingresos puede ser reflejada en un nuevo precio de la empresa objetivo.

\section{Tipos de ajuste de precio a la fecha de cierre.}

Los principales tipos de ajuste de precio - con corte, referencia o incidencia a eventos ocurridos a la fecha de cierre- en transacciones de M\&A son los siguientes:

\section{a) Ajuste sobre la base del capital de trabajo.}

Nos encontramos frente al mecanismo de ajuste de precio más utilizado, probablemente con el relacionado a los niveles de caja y deuda -explicado en el punto siguiente-. El capital de trabajo se define financieramente como la diferencia entre los activos corrientes y los pasivos corrientes de una empresa. Es decir, este concepto establece con relativa claridad la capacidad de una empresa de cumplir con sus deudas corrientes o de corto plazo.

La práctica estándar en materia transaccional es incluir en los contratos de adquisición que el precio ha sido establecido en base a un capital de trabajo objetivo o mínimo, con lo cual a través de la cláusula de ajuste de precio por capital de trabajo se protege al comprador de tener que hacer inversiones adicionales para cubrir las deudas que se pudieran generar en el día a día del negocio. Ahora bien, aunque no es usual, un vendedor podría entregar la compañía con un nivel superior al capital de trabajo pactado, con lo cual esta cláusula terminaría beneficiando al vendedor salvo que se pacte que cualquier ajuste de precio relacionado a este concepto solo beneficiará al comprador.

El procedimiento usual es el siguiente:

(i) Fijación de capital de trabajo mínimo u objetivo: Los asesores financieros del comprador y del vendedor deben fijar un monto de capi- tal de trabajo estimado al momento de firma del contrato, que será el capital de trabajo mínimo u objetivo que en base a sus proyecciones es el que requiere la compañía para operar. Es sumamente importante definir qué cuentas y conceptos serán considerados para el capital de trabajo. Incluso muchas veces se incluye como anexo un ejemplo de cálculo para claridad entre las partes.

La determinación del capital de trabajo mínimo u objetivo puede efectuarse en base a diversos factores, tales como el capital de trabajo histórico de la compañía objetivo, el capital de trabajo de compañías similares o incluso en base a un periodo determinado. Lo idea es que se el capital de trabajo mínimo u objetivo sea determinado a medida de la empresa objetivo ${ }^{14}$.

(ii) Comparación entre el capital de trabajo mínimo u objetivo y capital estimado a la fecha de cierre: El capital de trabajo mínimo u objetivo luego será comparado con el capital de trabajo estimado de la compañía objetivo a la fecha de cierre de la transacción. En base a ello, a la fecha de cierre se pagará un monto que puede ser mayor o menor al acordado, basándonos en la diferencia que podría existir entre el capital de trabajo mínimo u objetivo y el capital de trabajo estimado a la fecha de cierre. El monto del capital estimado a la fecha de cierre es proporcionado por el vendedor - usualmente el día anterior-, dado que sigue en control de la compañía y su equipo financiero monitorea la empresa objetivo.

(iii) Comparación entre el capital de trabajo estimado a la fecha de cierre y el capital de trabajo real: El capital de trabajo estimado a la fecha de cierre - que es sobre el que finalmente se calcula el precio a pagar en la fecha de cierre- luego será comparado con el capital de trabajo real a la fecha de

14. REBAZA, Alberto y OCHOA, Lucía. “Controversias principales en contratos de compraventa de acciones". En: Fusiones \& Adquisiciones. Lima: Instituto Pacífico, 2015, pp. 59-60. 
cierre de la transacción esta información se obtiene luego de la auditoría contable que realiza el comprador con posterioridad a la fecha de cierre, según se describe en la sección 4.4 (a). En base a ello se procederá a ajustar el precio en base a lo siguiente:

- Si el capital de trabajo real a la fecha de cierre es mayor que el capital de trabajo estimado a la fecha de cierre, se ajustará el precio a favor del vendedor.

- Si el capital de trabajo real a la fecha de cierre es menor que el capital de trabajo estimado a la fecha de cierre, se ajustará el precio a favor del comprador.

\section{b) Ajuste sobre la base de los montos de caja y deuda.}

Tomando en cuenta que, como hemos mencionado, el método de valorización más popular es el de flujo de caja descontado, no es sorpresa que este sea el método de ajuste más utilizado, conjuntamente con el ajuste sobre la base de capital de trabajo.

Cuando se utiliza el método de valorización descrito, el precio acordado entre las partes usualmente se pacta como libre de deuda neta, por lo que en teoría la empresa objetivo debería ser vendida en la fecha de cierre (i) sin deudas; $y$ (ii) sin efectivo en exceso. No obstante lo anterior, tomando en cuenta las dificultades que le genera al vendedor transferir una empresa en estas características en la práctica, lo usual es que se permita que el vendedor tenga cierto nivel de deuda financiera y de efectivo en la compañía objetivo - con determinados límites evidentemente, para darle certeza al comprador - con lo cual se (i) ajustará el precio a favor del comprador en caso la compañía tenga mayor deuda financiera a la estimada; $y$ (ii) a favor del vendedor en caso la entregue con mayor efectivo al estimado.
Como mencionamos en el párrafo anterior, es usual que este método de ajuste se utilice conjuntamente con el que se basa en capital de trabajo, con lo cual al monto resultante del ajuste por caja y deuda, debe sumarse el monto correspondiente a la deuda de capital de trabajo estimada y real a la fecha de cierre según se explica en el punto anterior.

\section{c) Ajuste sobre la base de los activos netos.}

Mediante una cláusula de ajuste sobre los activos netos, se comparará el monto resultante de la diferencia entre los activos netos y los pasivos netos de la empresa objetivo ${ }^{15}$ entre (i) la fecha de los estados financieros utilizados para la valorización; y (ii) la fecha de cierre.

Como puede inferirse, este tipo de ajuste es bastante utilizado cuando la empresa objetivo tiene activos con un alto nivel de rotación en el desarrollo de su actividad empresarial.

\section{d) Ajuste sobre la base de los ingresos de la empresa objetivo.}

A través de este mecanismo, se busca ajustar el precio utilizando como base los ingresos de la empresa objetivo, siendo el predilecto, el EBITDA. Como hemos visto, en algunos casos, el precio de una empresa objetivo se basa en el EBITDA a la fecha de los últimos estados financieros proporcionados, aplicándosele un múltiplo. Durante el periodo de análisis de ajuste de precio, se llegará al EBITDA final de la empresa objetivo, y en base a ello, se establecerá si el precio debe ser ajustado a favor del comprador -en caso el EBITDA final sea inferior al utilizado para definir el precio, llamado EBITDA estimado-, o a favor del vendedor - en caso el EBITDA final sea superior al EBITDA estimado-. EI mecanismo es similar al señalado para el ajuste por capital de trabajo.

\section{El caso especial del Locked Box.}


El mecanismo del locked box implica que el precio que el comprador pagará el vendedor en la fecha de cierre de la transacción ya ha sido acordado en base a estados financieros a una fecha determinada, conocida como el locked box date, no habiendo necesidad de aplicar un true-up mechanism o mecanismo de sinceramiento en el precio con posterioridad, tales como los descritos en el numeral 4.2 anterior.

Así, las partes acuerdan a una fecha determinada todos los aspectos relacionados a la proyección de caja, deuda y capital de trabajo entre la fecha del locked box y la fecha de cierre, acordando únicamente determinados eventos -objetivos y precisos- en los que se podrá modificar el precio, conocidos como leakages - acciones o eventos que resten valor a la empresa objetivo que ocurran desde el locked box date hasta la fecha de cierre-. Estos eventos usualmente incluyen la distribución de dividendos, pagos extraordinarios a la gerencia, bonos, financiamientos extraordinarios, entre otros.

A efectos de no trabar la marcha de la empresa, así como es común definir qué eventos serán considerados leakages, también se definirá el concepto de permitted leakages, relacionados a eventos que, si bien pueden restar valor a la compañía objetivo, son permitidos —usualmente ya se encuentran incluidos en la valorización del locked box al locked box date-.

Como podemos observar, el mecanismo del locked box es menos complicado que los métodos tradicionales de ajuste de precio. Como también se puede inferir, este mecanismo es primariamente favorable para el vendedor, ya que (i) a partir del locked box date transfiere los riesgos asociados al desarrollo del negocio al comprador; $y$, (ii) permite un exit rápido de la compañía objetivo a vendedores con ese perfil.

Ahora bien, no todo es malo para el comprador en este mecanismo, ya que, al tener un precio de compra definido, el planeamiento de la adquisición, financiamiento y eventuales autorizaciones serán más fáciles de obtener. El comprador deberá tener especial cuidado en realizar un due diligence financiero profundo para que las proyecciones sean lo más certeras posibles, y asegurar en el contrato de adquisición que en caso de leakages el comprador sea indemnizado desde el primer dólar por el comprador.

\section{Procedimiento.}

En corto, el procedimiento regular para realizar la revisión del ajuste de precio es el siguiente:

a) Dentro de un plazo que usualmente ronda entre 45 y 90 días calendario siguientes a la fecha de cierre, el comprador preparará a través de una empresa auditora de prestigio los cálculos relacionados a los conceptos relacionados a las cláusulas de ajuste pactadas -EBITDA final, el estado de situación financiera no auditado a la fecha de cierre, cálculos relacionados a las variaciones en la caja, deuda y capital de trabajo-, con un certificado de ajuste en base al método establecido por las partes.

b) Los documentos anteriores se preparan de acuerdo con los PCGA, y es usual pactar que se realicen en base a las mismas políticas, prácticas, procedimientos contables y métodos de valuación consistentes y aplicados en los estados financieros de la empresa objetivo.

c) El vendedor usualmente tendrá un plazo que oscila entre 30 y 45 días calendario para aceptar $\mathrm{u}$ objetar el ajuste propuesto por el comprador. En caso de discrepancia, lo usual es que un árbitro contable solucione la disputa.

d) El pago usualmente está garantizado a través de montos depositados en cuentas escrow, liberados por el agente escrow de acuerdo al resultado del ajuste.

\section{ESTRUCTURACIÓN DE CLÁUSULAS DE AJUSTE DE PRECIO POR HECHOS O EVENTOS POSTERIORES AL CIERRE: EARNOUTS}




\section{Concepto.}

A diferencia de las cláusulas de ajuste de precio descritas en la sección 4 anterior, los earnouts se utilizan como una forma de pago de compra en las adquisiciones donde existe cierta incertidumbre respecto de a los resultados que arrojará la empresa adquirida con posterioridad a la compra o cuando el comprador no tiene la certeza de que los resultados financieros del periodo revisado en el due diligence sean replicables o sostenibles en el tiempo. Esta forma de pago sujeta al cumplimiento de objetivos e hitos ayuda a cerrar la brecha entre la apreciación de la valorización de la empresa que puede existir entre el comprador y el vendedor, y así evitar que las partes se desanimen de llevar a cabo la transacción en base a dichas diferencias.

Imaginemos un supuesto en que vendedor y comprador no se ponen de acuerdo en el precio de la empresa objetivo, siendo la diferencia de perspectivas en el precio de USD 50 millones. Mediante un mecanismo de earnout, las partes pueden fijar un precio inicial y luego establecer el cumplimiento de hitos/objetivos para alcanzar e incluso superar el precio que el vendedor tenía en mente. Así, el vendedor estará en una posición en la que si bien no recibe el precio total que busca de manera inmediata en la fecha de cierre, tiene la posibilidad de obtenerlo, dependiendo de los resultados que obtenga la empresa objetivo en el futuro; mientras que el comprador sentirá que está adecuadamente protegido de pagar un sobreprecio compartiendo el riesgo del desarrollo del negocio con el vendedor.

Los asesores financieros del comprador desarrollarán el punto de equilibrio de pago del earnout contra los resultados a futuro, por lo cual necesariamente incluso pagando un precio mayor, seguirá beneficiándose en el futuro. De esta forma, en papel y práctica, se unifican los intereses del vendedor y los del comprador para la marcha adecuada del negocio.

Como es evidente, un vendedor tiene un interés directo y real respecto al cumplimiento de las provisiones contingentes ya que de dicho cumplimiento dependerá el pago de esta parte del precio. Dicho interés se traduce en el interés de participar en el negocio, alineándose con el interés del comprador de que el negocio adquirido funcione, previo análisis financiero del punto de equilibrio a partir del cual obtendrá rentabilidad y sobre el cual se comienza a negociar el pago del earnout.

\section{Estructuración.}

La clave para entender cómo estructurar una cláusula de earnout - así como su valorización — reside en verla análogamente como una especie de opción financiera. Desde un punto de vista racional, la única circunstancia en que una persona ejercería una opción es si el valor del activo subyacente excede el precio al que se adquirió la opción. En tal sentido, el valor que un inversionista recibe de una opción es "contingente" o depende de los resultados del activo subyacente.

Al igual que las opciones, los mecanismos de earnout se hacen "a medida" en situaciones de incertidumbre. Las opciones pueden generar mayor valor mientras más incierto o volátil es el activo subyacente. En el caso de los earnouts, mientras más incierto o difícil de predecir sea el futuro de la empresa objetivo, mayor rendimiento puede generarse. La situación actual del mercado y el atractivo de invertir en industrias riesgosas como tecnología, innovación, química y los fondeos a startups hacen de los earnouts un mecanismo ideal para alinear los intereses de las partes.

Desde nuestra perspectiva, los elementos esenciales que se deben considerar para estructurar de manera efectiva un mecanismo de earnout son los siguientes:

\subsection{Monto del earnout y métodos de valorización.}

Las partes deben determinar claramente qué parte del precio de venta será pagado al cierre de la transacción, y qué parte estará sujeta a la cláusula de earnout. En la práctica, cada una de ellas tratará de minimizar su riesgo en la adquisición:

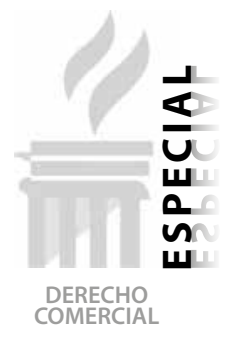

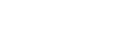


(i) Mientras el comprador tratará de incrementar el ratio del earnout ${ }^{16}$, (ii) el vendedor tratará de obtener más efectivo en la fecha de cierre.

La base para toda transacción es que las partes puedan ponerse de acuerdo en una parte del precio. El comprador y el vendedor saben que determinada empresa al menos vale una suma de dinero. Esta parte del precio se conoce como la porción "fija" o "no-contingente" del precio de venta que se pacta en el contrato de adquisición. Precisamente, el porcentaje del earnout se negocia en función a la diferencia en el precio percibido por las partes. La base para el earnout será la diferencia entre la parte "fija" del precio de venta y lo que el vendedor finalmente espera recibir como precio.

Mientras que los earnouts más simples pueden ser valorizados a través del método de flujo de caja descontado, los earnouts más complejos usualmente requieren que los asesores financieros utilicen métodos más sofisticados, como la simulación Monte Carlo para el caso de metas financieras o de rendimiento, o un análisis de opciones para earnouts con milestones no financieros.

Después de escoger el método de valorización, la tasa de descuento debe ser estimada a efectos de traer a valor presente los futuros pagos sujetos al earnout. Hay muchos factores asociados al riesgo y finalmente la volatilidad de los flujos de caja parece ser el determinante para obtener la tasa de descuento aplicable. Si hay certeza respecto al cumplimiento del earnout, la tasa de descuento a aplicar será equivalente, en la práctica, a la que se aplicaría a un financiamiento de compra con deuda, mientras que en supuestos de incertidumbre respecto al pago del earnout nos encontramos ante tasas de descuento cercanas a las de venture capital.

\subsection{Periodo del earnout.}

Dependerá mucho del tipo de negocio y tamaño de la transacción, pero por lo general, los mecanismos de earnout tienen un periodo que puede ir entre 1 y 10 años. El periodo usualmente es proporcional al ratio del earnout: A mayor ratio, mayor tiempo.

Bajo los conceptos financieros del valor del dinero en el tiempo, las partes ciertamente tendrán intereses opuestos respecto al periodo del earnout. El vendedor querrá periodos más cortos para incrementar el valor presente del pago; mientras que el comprador querrá extender los pagos del earnout por el mayor tiempo posible a efectos de reducir el valor presente de dichos pagos. Asimismo, desde un punto de vista no financiero necesariamente, un periodo mayor implicará que los gerentes claves e incluso los vendedores se mantengan más tiempo en la empresa objetivo, lo que generará un valor agregado para el comprador.

\subsection{Forma de pago.}

Evidentemente las cosas son más simples cuando las partes establecen que el precio contingente será pagado en efectivo. Las complejidades pueden presentarse cuando dicho pago incluye acciones del comprador.

Las partes deben considerar los aspectos tributarios del pago al definir si se utilizará efectivo, acciones o una mezcla de ambas formas de pago. El efectivo usualmente estará sujeto al pago de impuesto a la renta, por lo que en algunos casos el pago a través de acciones puede ser una buena alternativa dependiendo del interés de las partes.

El rol de los abogados es fundamental. Más allá de las cláusulas de protección por fluctuaciones en el precio de la acción -en caso el comprador cotice en alguna bolsa de valores - el vendedor debe negociar por anticipado un pacto de accionistas que lo proteja en su posición de accionista del comprador -en caso reciba acciones comunes-, incluyendo derechos de

16. Entendido como el porcentaje del precio máximo total que se le atribuirá al earnout en lugar del precio fijo pactado como precio de compra de la empresa. 
preferencia, de salida, derecho de tag-along, put options, derechos de información e incluso a nombramiento de directores dependiendo del porcentaje accionario que se adquiera.

\subsection{Tipos de objetivos y metas.}

Existen muchos criterios para poder cuantificar los resultados de una empresa. Para ser efectivos, las metas y objetivos deben estar claramente definidos, entendidos conjuntamente, obtenibles y cuantificables sin lugar a dudas. Los criterios usualmente utilizados por las partes para el pago del earnout generan una serie de problemas que deben ser acordados y definidos previamente para evitar conflictos. Si bien es cierto que estas metas usualmente tienen un carácter económico/financiero, también es usual que se incluyan hitos no financieros. A continuación, explicamos los principales tipos de objetivos y metas y los inconvenientes y consideraciones que pueden/deben tener las partes al momento de pactarlas:

a) Ingresos: Los earnouts basados en ingresos de la empresa objetivo son bastante comunes, aunque implican una serie de regulaciones respecto a la forma de medirlos. Este tipo de estructura funciona muy bien en los casos en que la gerencia de la empresa objetivo no pretende quedarse en la sociedad después de la transacción. En dichos escenarios, una cláusula de earnout provee a las partes de un mecanismo justo de valorizar el brand equity generado por los gerentes.

El principal riesgo que asume el comprador si utiliza esta estructura es que la gerencia de la empresa objetivo comience a vender sus productos a bajos márgenes, generando con esto un aumento considerable en los ingresos - y afectando directamente el EBITDA-. Un comprador diligente puede mitigar dicho riesgo pactando un monto mínimo de margen o estipulando que es necesario que la empresa objetivo tenga utilidad para llevar a cabo el earnout -lo cual es lógico desde un punto de vista comercial-.
Por su parte, el vendedor tratará de aprovechar la integración con el comprador en beneficio de la empresa objetivo, buscando que ponga a disposición recursos, activos para producción y canales de distribución con capacidad suficiente para cubrir la demanda del mercado por los productos de la empresa objetivo. Es recomendable pactar qué recursos se pondrán a disposición y no dejar una cláusula general de ayuda a la venta, a efectos de evitar conflictos respecto a la razonabilidad del soporte y la buena o mala fe del comprador.

b) Utilidad bruta: Una forma de reducir el riesgo anterior sería a través de un pacto en el cual la meta es un monto determinado de utilidad bruta de la empresa objetivo. Como uno puede imaginarse, pactar utilidad bruta como métrica también puede traer implicaciones que podrían generar controversia entre las partes en la ejecución del earnout. ¿Qué gastos deben aplicarse? ¿Quién tiene el control de la administración? ¿Qué tanta autonomía tiene el gerente financiero para definir qué aplicar como gastos generales?

La fórmula de earnout debe especificar cómo las partes determinan los gastos generales, políticas de sobrecostos, requisitos de compra y contratación, y los demás factores que la componen dependiendo del tipo de industria podrían afectar la utilidad bruta. La función del abogado será graficar dicha fórmula de la mejor manera en el contrato de adquisición.

c) EBITDA o flujos de caja: Si las partes han utilizado un múltiplo del EBITDA para valorizar la empresa objetivo al cierre, lo usual $-y$ conveniente- es establecer el cálculo de earnout de igual manera para resaltar la importancia de proveer flujos de caja -y por consecuencia, valor futuro- al comprador.

d) Milestones o metas concretas: Como mencionamos, los pagos del earnout también pueden tener un carácter no-financiero. Estas metas pueden incluir: cumplir con el desarrollo de determinado producto o pa-

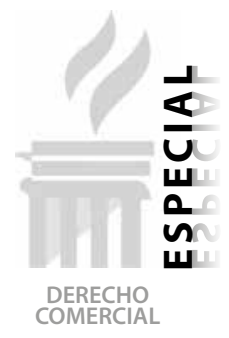

COMERCIA

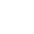


tente, llegar a un número mínimo de envíos o clientes, obtener la firma de un contrato importante con el Estado o un privado, la obtención de determinada licencia o autorización, entre otros.

Este tipo de objetivos se presenta mucho en los sectores de tecnología e innovación, así como en las industrias química y farmacéutica. En muchos casos en la compra de startups, independientemente de la industria, este tipo de metas funciona bastante bien, por la misma naturaleza y estado prematuro de la empresa objetivo.

Lo usual es incorporar una combinación de las 4 metas u objetivos anteriores en el criterio de la fórmula de earnout. En la práctica se presentan fórmulas de cumplimiento conjunto, así como cláusulas que le otorgan un peso determinado a cada objetivo, lo que permite que cada uno de estos pueda ser alcanzado de manera independiente. A manera de ejemplo, las partes podrían pactar que el pago anual dependa $40 \%$ del EBITDA, $40 \%$ de los ingresos y un $20 \%$ si se consigue registrar una patente nueva de determinadas características o si el área de $R \& D$ logra desarrollar un prototipo o producto nuevo.

Un estudio sobre earnouts publicado en The Chicago University Press Journals concluyó que, en promedio, los vendedores reciben alrededor del $62 \%$ del monto total de precio contingente, y en un $42 \%$ de los casos, el $100 \%$ del mismo ${ }^{17}$.

Una vez que las partes han acordado cuáles serán los objetivos que se utilizarán para el earnout, así como las reglas contables y financieras aplicables, se debe decidir cómo se realizarán los pagos. En la práctica, es usual es que el cumplimiento de los objetivos se revise de manera anual.

\subsection{Cronograma de pagos.}

Hay muchas opciones en las que se puede estructurar un cronograma de pagos para una compra a través de un mecanismo de earnout. Lo usual es que el riesgo del cumplimiento de los objetivos sea asignado de manera balanceada entre el comprador y el vendedor, de manera que el cumplimiento parcial del objetivo también sea recompensado con parte del pago. Imaginemos el siguiente supuesto: El objetivo pactado en el earnout para el pago del monto anual fue obtener un EBITDA de USD 10 millones. Al final del año, la empresa arroja un EBITDA de USD 9 millones. Lo usual en estos casos es que el pago sea prorrateado dependiendo del porcentaje del objetivo alcanzado.

Las posibilidades son amplias y lo que se pacte finalmente dependerá de la industria y del interés de las partes. En vez de prorratear, se puede pactar un cronograma de pagos separado en rangos, con milestones, dependiendo del cálculo financiero de los puntos de equilibrio para el comprador.

El cronograma de pagos también debe establecer qué sucede cuando el vendedor logra que la compañía objetivo exceda los objetivos planteados. La práctica respecto a este punto es variada. Se puede establecer bonos, pago de un precio mayor o incluso la posibilidad de aplicar dicho exceso al siguiente periodo del earnout para llegar al cumplimiento de las metas anuales - aunque indefectiblemente en este último caso, para el último año se deberá cuantificar cualquier exceso para la materialización en un pago en efectivo o acciones-. Incluso en algunos casos se establece una suma alzada para el final del periodo del earnout a pagarse contra el cumplimiento acumulado de las metas anuales.

El comprador, a efectos de minimizar o cubrir su riesgo y la impredictibilidad del mercado usualmente establece caps en los pagos que deba hacer como consecuencia del rendimiento de la empresa objetivo en el periodo de earnout. El

17. KOHERS, Ninon y ANG James. "Earnouts in Mergers: Agreeing to Disagree and Agreeing to Stay". En: The University of Chicago Press Journals. The Journal of Business, Vol. LXXIII, N 3 . Chicago: 2000, pp. 445-476. 
vendedor por su parte, en el supuesto anterior, buscará que se paguen montos mínimos establecidos por año, así como bonos por el cumplimiento de las metas de la empresa objetivo.

\subsection{Integración operacional.}

Un aspecto fundamental a considerar al momento de estructurar un earnout es el nivel de participación que un comprador tendrá en las operaciones de la empresa objetivo. El contrato debe definir claramente qué unidad o línea del negocio es la que se considerará para efectos de cumplir con los objetivos y quién tendrá el control de los aspectos corporativos importantes de dicha unidad de negocios. En muchos casos el motivo de la adquisición es realizar una integración de líneas de negocio, productos u operaciones, con lo cual al negociar el precio y encontrarse las partes frente a un mecanismo de earnout se debe considerar quién tendrá la decisión sobre las operaciones integradas.

Un par de supuestos pueden revelar la complejidad que puede ocasionar la integración de las partes:

(i) Si el comprador dentro de su estructura comercial para la adquisición pensó en incluir una gama de sus productos en la empresa objetivo para que esta los administre, produzca o venda, ¿los ingresos y gastos relacionados a dicha operación conjunta a quién deben ser asignados? ¿Quién supervisa la razonabilidad y división de esas operaciones que - precisamente- fueron concebidas como conjuntas al evaluar la adquisición?

(ii) Si la razón de la adquisición para el comprador fue realizar una integración vertical para una línea de su negocio, y es el principal comprador de la empresa objetivo, ¿qué pasa con la diferencia de valor que podría existir entre el valor de venta al compradory el valor de venta que podría obtener de terceros? Las reglas de precio de transferencia no llegan a cubrir totalmente esta situación, por lo que se tendría que pactar claramente entre las partes qué sucede con esa eventual diferencia - ¿Se cuenta ficticiamente como ingreso? ¿La asume el vendedor directamente o a través de un bono?-.

Si el vendedor se queda con el control operacional de la empresa objetivo es menos probable que pueda argumentar que por interferencia del comprador no pudo cumplir con los objetivos pactados en el earnout. Como se puede inferir, mientras menos integración operacional por parte del comprador se produzca, más efectivos y fáciles de comprobar serán las métricas del earnout. El interés del vendedor será claro: a efectos de cumplir con los objetivos planteados querrá mantener el control operacional de la empresa objetivo, mientras que el interés del comprador será beneficiarse de la empresa objetivo - sino la adquisición no tendría lógica en primer lugar- con lo cual los intereses de las partes si bien económicamente confluyen, se contraponen operacionalmente.

Para solucionar esta disociación de intereses operacionales, usualmente es importante ubicar cuál es el área de interés operacional para el comprador y sobre ese punto pactar el cumplimiento de los objetivos y resultados del earnout en base al nivel de integración y capacidad en la toma de decisiones que requiere el comprador. De manera alternativa, se puede pactar un periodo agresivo de earnout con lo cual el periodo de integración posterior puede manejarse en base a las expectativas del comprador. En conclusión, el earnout debe estructurarse de tal forma que permita la consecución del plan estratégico y comercial de la empresa, mientras a su vez se obtienen las metas financieras y no-financieras del earnout. Esto claramente es más fácil de lograr en la teoría que en la práctica.

\section{CONSIDERACIONES ADICIONALES}

Habiendo analizado ambos tipos de cláusula de ajuste de precio, recomendamos que, en adición a los aspectos específicos descritos, se tomen en cuenta las siguientes consideraciones generales:

\section{Eventual duplicidad con las representaciones y garantías en una cláusula de ajuste de precio.}

Considerando que casi con seguridad el contrato de adquisición contendrá una cláusula que

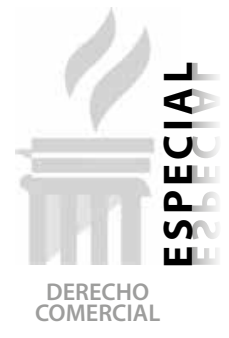

COMERCIAL 
contenga declaraciones y garantías sobre el contenido y preparación de los estados financieros, se debe tener cuidado en no aplicar una doble penalidad en caso que como consecuencia de la auditoría posterior al cierre, se verifique que corresponde un ajuste de precio en relación a los estados financieros analizados para la valorización. A efectos de evitar una doble sanción para el vendedor -indemnización por falsedad o inexactitud de la representación y garantíassumada a la"penalidad" de ver ajustado el precio, es recomendable incluir una cláusula de "no duplicidad" en el contrato de adquisición.

\section{Garantía del pago.}

Es usual que las obligaciones relacionadas al ajuste de precio se encuentren garantizadas a través de montos depositados en cuentas escrow. Así, cuando se verifique el cumplimiento de las condiciones propuestas para el ajuste, el agente escrow simplemente liberará los fondos a favor de la parte que corresponda según las instrucciones establecidas en el contrato escrow.

\section{Definición de conceptos.}

Los conceptos que se incluyan en la sección de definiciones del contrato de adquisición deben ser precisos y trabajados de manera conjunta entre asesores legales, contables y financieros a efectos de que realmente reflejen el contenido deseado y aplicable a los supuestos de ajuste de precio que se busca regular.

\section{Mecanismo de solución de controversias.}

Considerando la cantidad de discrepancias que podrían generarse en la aplicación de los conceptos desarrollados en el presente artículo, y que, en adición, muy probablemente impliquen una sensibilidad especial al afectar directamente la situación económica de las partes, es sumamente importante establecer un mecanismo de solución de controversias claro y con entes sofisticados que permitan a las partes tener certeza de una solución rápida y efectiva. A tales efectos, se sugiere pactar una terna de árbitros contables reputados de la cual las partes puedan seleccionar al decisor, así como los alcances y plazo máximo de revisión para un pronunciamiento.

\section{CONCLUSIONES}

La volatilidad e incertidumbre del mercado han demostrado empíricamente que es muy difícil determinar el valor exacto de una empresa involucrada en un proceso de adquisición. Aspectos culturales, financieros, de aversión al riesgo y operacionales hacen complicado que vendedor y comprador acuerden una valorización de la empresa objetivo de manera rápida $y$, por lo tanto, un precio fijo para la adquisición. Esta brecha puede generarse por incertidumbre, por selección adversa o por otras razones, pero la realidad es que es una constante en los procesos de fusiones y adquisiciones.

Las cláusulas de ajuste de precio pueden proteger a compradores y vendedores para establecer un valor real de la empresa a definirse en un futuro cercano - cláusula de ajuste al cierre-o en un horizonte algo más lejano - earnouts-, sin dejar de cerrar la transacción en el momento necesario para comprador y vendedor, fomentando así el movimiento de capitales.

Como hemos podido observar al analizar los aspectos a considerar al estructurar una cláusula de ajuste de precio, si bien los asesores comerciales y financieros del comprador tienen una dura tarea definiendo las fórmulas, ratios, porcentajes, valorizaciones y modelos financieros, la labor del abogado no deja de ser menos compleja. Los abogados transaccionales tenemos que saber qué complejidades engloba la decisión de implementar cláusulas de ajuste de precio y estar familiarizados con los términos financieros, así como con las consecuencias legales y operativas de sujetar una parte del precioa un mecanismo de earnout, ya que implicará prever de la mejor manera los escenarios probables en que las partes pueden tener desencuentros para que el proceso de earnout se lleve de manera fluida, reduciendo - al menos - las controversias posteriores al cierre. En todo caso, estas cláusulas logran que compradory vendedor sientan que el precio al que se adquirió y vendió fue el precio justo en base a la información financiera histórica y/o proyecciones y resultados de la empresa a futuro. 